\title{
Pyruvate kinase type M2: a crossroad in the tumor metabolome
}

\author{
S. Mazurek ${ }^{1}$, H. Grimm ${ }^{2}$, C. B. Boschek ${ }^{3}$, P. Vaupel $^{4}$ and E. Eigenbrodt ${ }^{1} *$ \\ ${ }^{1}$ Institute for Biochemistry \& Endocrinology, Veterinary Faculty, University of Giessen, Frankfurter Strasse 100, 35392 \\ Giessen, Germany \\ ${ }^{2}$ Centre of Surgery, Medical Faculty, University of Giessen, Klinikstrasse 29, 35392 Giessen, Germany \\ ${ }^{3}$ Institute for Medical Virology, Medical Faculty, University of Giessen, Frankfurter Strasse 107, 35392 Giessen, Germany \\ ${ }^{4}$ Institute of Physiology and Pathophysiology, Medical Faculty, University of Mainz, Duesbergweg 6, 55099 Mainz, \\ Germany
}

\begin{abstract}
Cell proliferation is a process that consumes large amounts of energy. A reduction in the nutrient supply can lead to cell death by ATP depletion, if cell proliferation is not limited. A key sensor for this regulation is the glycolytic enzyme pyruvate kinase, which determines whether glucose carbons are channelled to synthetic processes or used for glycolytic energy production. In unicellular organisms pyruvate kinase is regulated by ATP, ADP and AMP, by ribose 5-P, the precursor of the nucleic acid synthesis, and by the glycolytic intermediate fructose $1,6-\mathrm{P}_{2}$ (FBP), thereby adapting cell proliferation to nutrient supply. The mammalian pyruvate kinase isoenzyme type M2 (M2-PK) displays the same kinetic properties as the pyruvate kinase enzyme from unicellular organisms. The mammalian M2-PK isoenzyme can switch between a less active dimeric form and a highly active tetrameric form which regulates the channeling of glucose carbons either to synthetic processes (dimeric form) or to glycolytic energy production (tetrameric form). Tumor cells are usually characterized by a high amount of the dimeric form leading to a strong accumulation of all glycolytic phosphometabolites above pyruvate kinase. The tetramer-dimer ratio is regulated by ATP, FBP and serine and by direct interactions with different oncoproteins (pp60 $\left.{ }^{\mathrm{v}-\mathrm{src}}, \mathrm{HPV}-16 \mathrm{E} 7\right)$. In solid tumors with sufficient oxygen supply pyruvate is supplied by glutaminolysis. Pyruvate produced in glycolysis and glutaminolysis is used for the synthesis of lactate, glutamate and fatty acids thereby releasing the hydrogen produced in the glycolytic glyceraldehyde 3-phosphate dehydrogenase reaction.
\end{abstract}

Immune cells: Nutrients: Serinolysis: Fatty acids

\section{Pyruvate kinase, the central nutrient supply sensor in unicellular organisms}

Cell proliferation is a process that consumes large amounts of energy. A reduction of the nutrient supply leads to cell death if cell proliferation is not limited (Mazurek et al. 1997a, 1999a; Shim et al. 1998; Durante et al. 1999). Therefore, unicellular organisms have a variety of sensoring mechanisms to adapt cell proliferation rate to variations in nutrient supply (Thevelein \& Hohmann, 1995; Teusink et al. 1998). One key sensor of this regulation is the glycolytic enzyme pyruvate kinase (E.C. 2.7.1.40). Pyruvate kinase is responsible for the transfer of phosphate from phosphoenolpyruvate to ADP accompanied by formation of ATP and pyruvate. Phosphoenolpyruvate, the substrate of pyruvate kinase is the most energy rich cellular phosphometabolite $\left(\Delta \mathrm{G}^{0}=58 \mathrm{~kJ}\right)$. In comparison, the $\Delta \mathrm{G}^{0}$ of ATP is $29 \mathrm{~kJ}$. Therefore, the pyruvate kinase reaction strongly favors the formation of ATP. The Kequilibrium (([PEP].([ADP]): ([pyruvate]·[ATP $]))$ is 1:2000 (pH 8.0, 30 $\left.{ }^{\circ} \mathrm{C}\right)$ (Hess, 1963; Valle et al. 1996). Therefore, energy production by pyruvate kinase is more effective than ATP production via mitochondrial respiration. Glycolytic energy production via pyruvate kinase can strongly suppress mitochondrial energy production (Gosalvez et al. 1974; Melo et al. 1998).

For this reason and since phosphoenolpyruvate and glycolytic phosphometabolites are precursors for synthetic processes, the pyruvate kinase mass action ratio is far removed from its Kequilibrium in all organisms (Hess, 1963; Mazurek et al. 1997a, 2001; Zwerschke et al. 1999; Bond et al. 2000). In unicellular organisms pyruvate kinase is strongly inhibited by ATP and activated by the energy sensor AMP, $\mathrm{P}_{\mathrm{i}}$ and ribose 5-P, the precursors of nucleic

Abbreviations: FBP, fructose 1,6-bisphosphate; M2-PK, pyruvate kinase type M2.

* Corresponding author: Dr E. Eigenbrodt, fax: +49 64199 38179, email Erich.Eigenbrodt@ vetmed.uni-giessen.de 


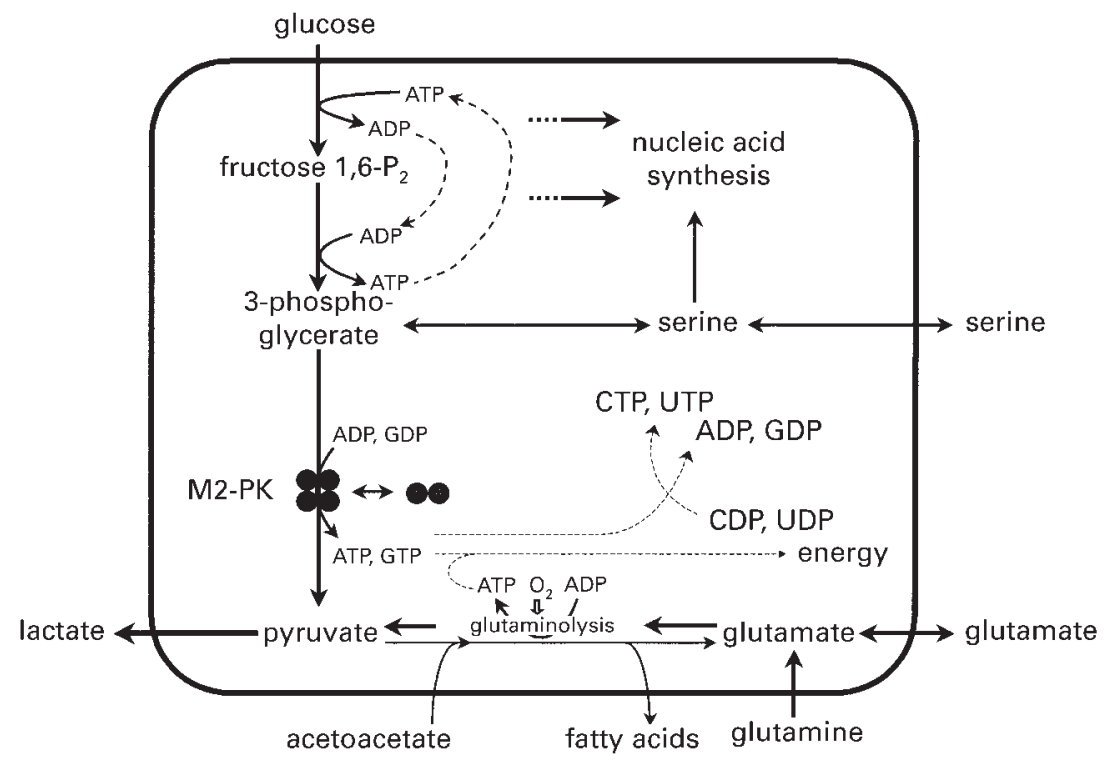

Fig. 1. Regulation of the ATP:ADP and GTP:GDP ratios in tumor cells.

acid synthesis (Fig. 1) (Atkinson \& Fall, 1967; Atkinson \& Walton, 1967; Maeba \& Sanwal, 1968; Tuominen \& Bernlohr, 1971; Schramm et al. 2000). By this mechanism, pyruvate kinase links energy rich metabolites to the flow of glucose carbons to nucleic acid synthesis, thereby stabilizing the so-called energy charge (([ATP]+1/2[ADP]): $([\mathrm{AMP}]+[\mathrm{ADP}]+[\mathrm{ATP}]))($ Atkinson \& Fall, 1967; Atkinson \& Walton, 1967). Phosphoenolpyruvate is a precursor of amino acids and complex carbohydrates such as sialic acid (Mazurek et al. 1997a). In addition, pyruvate kinase activity is modulated by carbamoyl-P and several different amino acids (Tuominen \& Bernlohr, 1971).

The inhibition of pyruvate kinase is essential for synthetic processes and for respiration. Inhibition of pyruvate kinase is highly dangerous when excess carbohydrates (sugars) are available. Glycolysis is organized in such a manner that at first two ATP are consumed for the synthesis of fructose 1,6-bisphosphate before ATP can be regained by the phosphoglycerate kinase (E.C. 2.7.2.3) reaction. Net ATP production occurs in the pyruvate kinase reaction (Thomas \& Fell, 1998). In addition, in the glycolytic glyceraldehyde 3-phosphate dehydrogenase reaction NADH is produced. The hydrogen is transferred to pyruvate, the product of the pyruvate kinase reaction and is released as lactate (Eigenbrodt $e t$ al. 1998a). Thereby, a constant inhibition of pyruvate kinase that favors synthetic processes, can lead to ATP depletion under both conditions: high and low carbohydrate supply (Thevelein \& Hohmann, 1995; Mazurek et al. 1997b, 1999a; Teusink et al. 1998). With an abundance of carbohydrates, ATP is completely consumed for the synthesis of fructose 1,6-bisphosphate. Since unicellular organisms are always in danger of variations in the carbohydrate supply, they have developed a special mechanism to prevent ATP depletion (Thevelein \& Hohmann, 1995; Teusink et al. 1998). The uptake of carbohydrates from the environment is fueled by phosphoenolpyruvate, the substrate of the pyruvate kinase reaction. Furthermore, pyruvate kinase is activated by sugar phosphates, especially fructose 1,6-bisphosphate that also regulates the glucose transporter (Thevelein \& Hohmann, 1995; Luesink et al. 1999). In accordance, in yeast cells, the overexpression of pyruvate kinase leads to an inhibition of cell proliferation by depletion of glycolytic phosphometabolites. The overexpression of the protein kinase MCKinase that phosphorylates and inactivates pyruvate kinase restores cell proliferation. Besides the inhibition of cell proliferation, the overexpression of pyruvate kinase also blocks the formation of spores (Brazill et al. 1997). Spores allow the survival of unicellular organisms under nutrient limitation and unfavourable external conditions for a longer time. Sporulation is linked to another phosphometabolite, 3-phosphoglycerate, which is accumulated in spores in a great amount (Nelson \& Kornberg, 1970a, $b$; Rhaese et al. 1976). In contrast to ATP, fructose 1,6-bisphosphate and phosphoenolpyruvate, 3-phosphoglycerate is highly stable but a low energy compound of the glycolytic sequence. However, under the catalysis of phosphoglyceromutase (E.C. 2.7.5.3) and enolase (E.C. 4.2.1.11), 3-phosphoglycerate is converted to energy rich phosphoenolpyruvate, the substrate of the ATP producing pyruvate kinase. During resporulation the first event is the activation of these three enzymes and the upregulation of the flow of 3-phosphoglycerate to pyruvate to restore NTP pools (Nelson \& Kornberg, 1970a, $b$ ). By linking energy metabolism with synthetic (anabolic) processes, pyruvate kinase dependent on nutrient supply, regulates cell proliferation, sporulation and cell death.

In recent years it has been shown that the same central role of pyruvate kinase in concert with other glycolytic enzymes is found in multicellular organisms, where nutrient supply is more or less constant. Studies with mammals have shown that also in these species, pyruvate kinase is involved in fundamental processes such as cell proliferation, differentiation, tumor formation and apoptosis (Mazurek et al. 1997a, 1999a). 


\section{The role of pyruvate kinase isoenzymes in multicellular organisms}

In multicellular organisms such as mammals only tumor cells and spermatides have comparably high levels of glycolytic phosphometabolites to those found in active proliferating unicellular organisms (Boscá \& Corredor, 1984; Eigenbrodt et al. 1985). In differentiated tissues phosphometabolite levels are 100-fold lower than in proliferating cells. One exception is glycerate 2,3-bisphosphate which is low in tumor cells and high in differentiated tissues, especially erythrocytes. In normal proliferating cells glycolytic phosphometabolites rise 10-fold in the G1-phase, but never reach levels as high as in tumor cells (Chesney et al. 1999; Aulwurm \& Brand, 2000; Perez et al. 2000). The isoenzyme of pyruvate kinase that allows the upregulation of phosphometabolite pools in multicellular organisms is pyruvate kinase type M2 (M2-PK). M2-PK is an ancient variant of the pyruvate kinase enzyme found in unicellular organisms such as yeast and E. coli (Eigenbrodt et al. 1985, 1992; Valle et al. 1996; Bond et al. 2000; Schramm et al. 2000). Since M2-PK is found in tissues with a high capacity for nucleic acid synthesis, we have termed those tissues nucleogenic tissues to differentiate them from gluconeogenic tissues with a high rate of gluconeogenesis and from lipogenic tissues with a high rate of lipogenesis (Mazurek et al. 1997a).

In differentiated tissues pyruvate kinase isoenzymes other than M2-PK are found: M1-PK is characteristic for tissues with high rates of energy consumption and low phosphometabolite pools such as muscle and brain. Pyruvate kinase type $\mathrm{L}$ is found in gluconeogenic tissues such as liver and kidney. In erythrocytes pyruvate kinase type $\mathrm{R}$ is expressed. The different pyruvate kinase isoenzymes react to individual allosteric regulators (Eigenbrodt et al. 1994). They are phosphorylated by different specific proteinkinases and are associated with different other enzymes as well as proteins with nonglycolytic functions such as actin, tubulin or band 3 protein (Eigenbrodt et al. 1994). The regulation of pyruvate kinase activities by association with other enzymes and proteins seems to be a very ancient phenomenon, since in bacteria the E-ras protein and nucleotide dikinase selectively bind to pyruvate kinase thereby modulating the ATP:ADP and GTP:GDP ratios (Chopade et al. 1997). Associations of different glycolytic enzymes with other proteins have also been found in mammalian cells. To demonstrate the association between glycolytic enzymes and other proteins different methods are used, e.g. coimmunoprecipitation and the two-hybrid technique (Zwerschke et al. 1999). We have developed a method to determine the association of several glycolytic enzymes and could show that not only the glycolytic enzymes but also RNA, oncoproteins and components of the proteinkinase cascade can be associated in a so-called glycolytic enzyme complex (Mazurek et al. 1996, 1998, 1999b, 2001). Demonstration of the glycolytic enzyme complex is based on the isoelectric points of the proteins. Proteins associated within the glycolytic enzyme complex focus at a common isoelectric point that is different from the isoelectric point of the purified individual proteins. Migrations of proteins inside and outside the glycolytic enzyme complex are reflected by shifts in their isoelectric points. The glycolytic enzyme complex has mainly been found perinuclear but some of the enzymes are also bound to the cytoskeleton, mitochondria or in the nucleus (Mazurek et al. 1997a, $b$; Bereiter-Hahn et al. 1998; Engel et al. 1998; Popanda et al. 1998; Nguyen et al. 2000). The association of enzymes and other compounds within the glycolytic enzyme complex leads to a compartmentalization of metabolic processes. The enzyme composition of the glycolytic enzyme complex controls phosphometabolite pools and the interaction between glycolysis, glutaminolysis and serinolysis (Mazurek et al. 2001).

M2-PK occurs in a highly active tetrameric form with a high affinity to its substrate phosphoenolpyruvate and in a less active dimeric form with a low PEP affinity. Only the tetrameric form of M2-PK is associated within the glycolytic enzyme complex (Mazurek et al. 1997b; Zwerschke et al. 1999).

During tumorigenesis the tissue specific isoenzyme generally disappears and M2-PK is expressed. In tumor cells generally the dimeric form of M2-PK is dominant and is released into the blood (Oremek et al. 1999). Therefore, we have termed the dimeric form of M2-PK as tumor M2-PK (Eigenbrodt et al. 1992). The dissociation of the tetrameric form to the dimeric form is induced by oncoproteins. The first oncoprotein that was found to interact with M2-PK was the activated $\mathrm{pp} 60^{\mathrm{v}-\mathrm{src}}$ kinase. The pp60 $0^{\mathrm{v}-\mathrm{src}}$ kinase phosphorylates M2-PK on a tyrosine residue (Presek et al. 1988; Eigenbrodt, et al. 1998b). Another oncoprotein that leads to a dimerization of M2-PK is the HPV-16 E7 oncoprotein that directly binds to M2-PK. The interaction of the oncoproteins with M2-PK always leads to an accumulation of all phosphometabolites above pyruvate kinase which are then channeled to synthetic processes, e.g. nucleic acid synthesis.

Besides the expansion of phosphometabolite pools, the dimerization of M2-PK has another important consequence that concerns energy metabolism. As in unicellular organisms, in mammals both glucose limitation and oversupply of glucose, lead to imbalances in the ATP:ADP ratio, to an inhibition of pyruvate kinase and can cause cell death (Live \& Kaminskas, 1975; Mazurek et al. 1997a, b, 1999a; Wang \& Iynedjian, 1997). Therefore, tumors with a high glycolytic flux rate are sensitive to glucose limitations (Mazurek et al. 1997b; Shim et al. 1998). Presumably, to prevent cell death by glucose limitation tumor cells have reduced mitochondrial pyruvate consumption and have activated a pathway that produces pyruvate and energy from the amino acid glutamine. This pathway has been termed as glutaminolysis in analogy to glycolysis, see Fig. 1 (McKeehan, 1982). In contrast to glycolysis, glutaminolysis is dependent upon oxygen supply (Eigenbrodt et al. 1998a). Both pathways are active in proliferating and lactating mouse mammary gland where the synthetic products of glycolysis such as fructose 1,6-bisphosphate and nucleotides are released into the milk. During mouse mammary gland apoptosis both pathways are simultaneously inactivated which leads to a drastic drop in the nucleotide triphosphate pools (Mazurek et al. 1999a). 
Investigations on human tumors xenografted in nude rats have revealed that besides glutamine the amino acid serine is used in great amounts as a substrate for pyruvate production. Serine is converted to 3-phosphoglycerate and further converted to pyruvate and lactate with the formation of ATP. This pathway has been termed serinolysis in analogy to glycolysis and glutaminolysis (Eigenbrodt et al. 1998a; Mazurek et al. 2001). In addition, serine can expand pyruvate pools when the flow through the glyceraldehyde 3-phosphate dehydrogenase (E.C. 1.2.1.12) reaction is limited. In solid tumors the flow of serine to lactate is highest when the glucose carbons are predominantly used for nucleic acid synthesis. Investigations of transformed NIH 3T3 cells revealed that the organization of the glycolytic enzyme complex plays an important regulatory role in this process. Only the tetrameric form of M2-PK is associated within the glycolytic enzyme complex. The dimeric form is located outside the complex. Transformation of a low glycolytic NIH 3T3 cell line which is characterized by a large amount of the dimeric form of M2-PK, high phosphometabolite levels and a high proliferation rate is only possible when phosphoglyceromutase reintegrates into the glycolytic enzyme complex (Mazurek et al. 2001). Thereby, the flow of serine to 3-phosphoglycerate, pyruvate and lactate increases. The glycolytic enzyme complex associated form of phosphoglyceromutase is the histidine phosphorylated form that is independent of glycerate 2,3-bisphosphate. The phosphorylation and activation of phosphoglyceromutase is presumably caused by a direct transfer of phosphate from the histidine of the enzyme nucleotide diphosphate kinase to the histidine of phosphoglyceromutase. This might be an explanation of why nucleotide diphosphate kinase is constantly altered during tumorigenesis (Mazurek et al. 1998).

\section{Fatty acids and glutamate, two alternatives for hydrogen release}

Due to an upregulation of glycolytic phosphometabolites, tumor cells usually contain no active glycerol 3-phosphate shuttle or only very low activities of glycerol 3-phosphate dehydrogenase (E.C. 1.1.1.8). Therefore, in tumor cells the hydrogen produced in the glycolytic glyceraldehyde 3-phosphate dehydrogenase reaction is transferred to pyruvate and released as lactate. In addition, tumor cells use two further pathways to export the hydrogen (Mazurek et al. 1997b).

Investigations with tumor cells and solid tumors have shown that tumor cells have the enzymatic and metabolic capacity for de novo synthesis of all fatty acids, whereby saturated and monounsaturated fatty acids are predominantly formed and released (Fig. 2) (Punnonen et al. 1989; Pizer et al. 1998). This explains why in contrast to normal tissues the membrane of tumor cells predominantly contains saturated and monounsaturated fatty acids (Punnonen et al. 1989; Lee et al. 1995). For de novo synthesis of fatty acids, acetyl CoA and NADPH are necessary. NADPH is produced in large amounts by the $\mathrm{NADP}^{+}$dependent malate decarboxylase (E.C. 1.1.1.40) and the $\mathrm{NADP}^{+}$dependent isocitrate dehydrogenase (E.C. 1.1.1.42). Both are strongly overexpressed in human tumors (Mazurek et al. 2000). The oxidative pentose phosphate cycle, the alternative NADPH producing pathway, is blocked by the high fructose 1,6-bisphosphate levels found in tumor cells. Therefore, in tumor cells the mass of ribose 5-phosphate is synthesized by the transketolase-transaldolase reactions (Boros et al. 1997; Lee et al. 1998).

In solid tumors, acetyl CoA derives from ketone bodies (acetoacetate and $\beta$-hydroxybutyrate) and glucose carbons. A good correlation was found between glucose consumption and

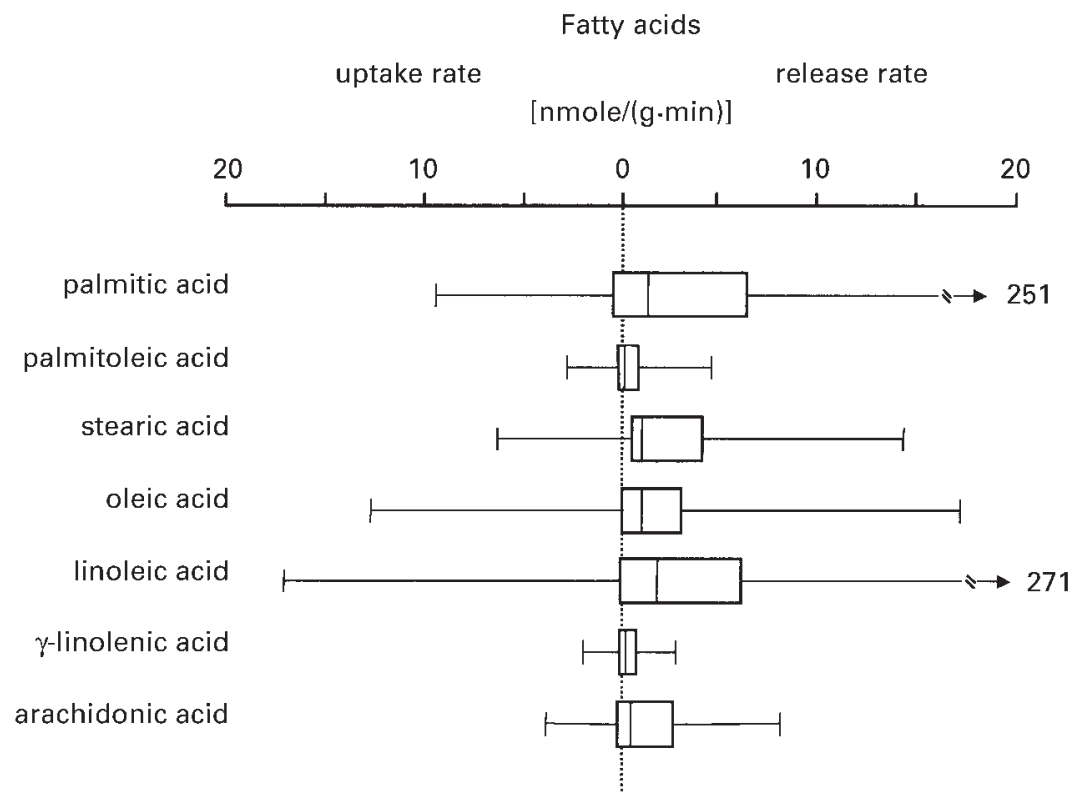

Fig. 2. Fatty acid conversion rate in xenotransplanted human mammary carcinomas, $n=66$. 
Interaction between

glucose uptake rate and fatty acid release rate

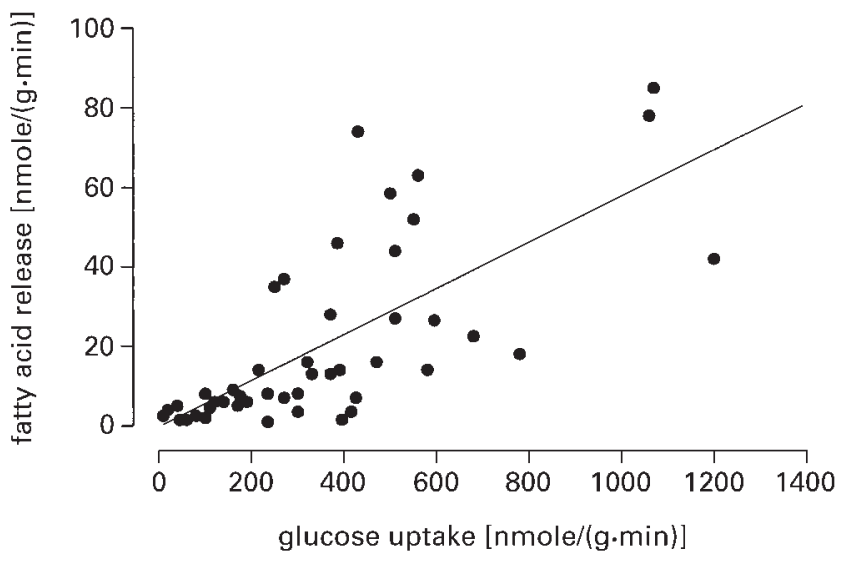

Fig. 3. Interaction between glucose uptake rate and fatty acid release rate in xenotransplanted human mammary carcinoma. Slope $=58$, intercept $=-0.25 \mathrm{nmole} /(\mathrm{g} \cdot \mathrm{min}), \mathrm{r}=0.706$. lactate production by tumors and between glucose consumption and fatty acid release rate (Fig. 3) (Kallinowski et al. 1987, 1988; Vaupel et al. 1987; Eigenbrodt et al. 1998a). In the mitochondria oxaloacetate and acetyl CoA condense to form citrate that is transported into the cytosol. In the cytosol citrate can be converted to glutamate and is released as glutamate or glutamine (Fig. 4). A greater part is converted to acetyl CoA and oxaloacetate by ATP citrate lyase. Oxaloacetate is further converted to malate and pyruvate by malate dehydrogenase and the $\mathrm{NADP}^{+}$dependent malate decarboxylase. Acetyl CoA and the NADPH produced in the malate decarboxylase reaction are channeled to fatty acid synthesis (Fig. 4). The fatty acids produced can be used for phospholipid synthesis or can be released from the cell. Glutamate, alanine, glycine and fatty acid released from tumors are able to suppress immune cell functions (Eck et al. 1989; Grimm et al. 1994, 1995; Jiang et al. 1998; Wheeler et al. 1999). Part of the acetate for the synthesis of glutamate derives from ketone bodies. Glutamate can be converted to glucose in liver and kidney. This may explain why tumor patients become cachectic without developing

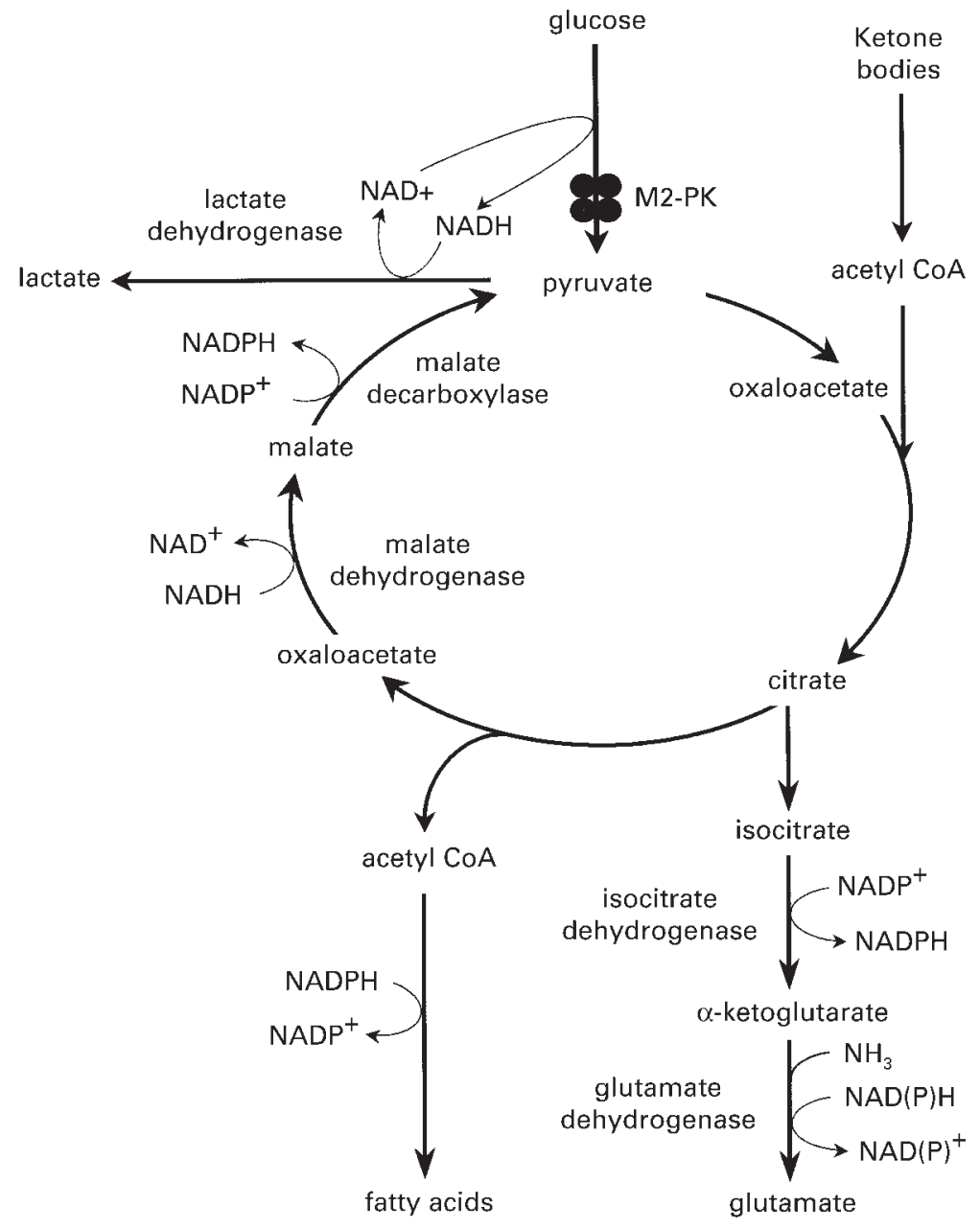

Fig. 4. Hydrogen release in tumor cells. In tumor cells one important way to release the hydrogen produced in the glycolytic glyceraldehyde 3-phosphate dehydrogenase reaction is the transfer to pyruvate and the release as lactate. Alternatively, the hydrogen can also be exported as glutamate or as fatty acids. 
hypoglycaemia or ketosis. Saturated and monounsaturated fatty acids inhibit 6-phosphofructo 1-kinase and M2-PK (Marchut et al. 1986). Therefore, in the future it will be interesting to learn whether those fatty acids can inhibit tumor growth by interfering with glycolysis.

Tumor cells and immune cells are characterized by similar metabolic features such as high glycolytic and glutaminolytic flux rates which allow them to invade areas with low levels of oxygen and glucose. It will therefore be very important to develop new immunotherapeutical approaches to learn more about the metabolic interference of both cell types (Sebolt \& Weber 1984; Brand et al. 1987; Newsholme \& Calder, 1997; Kew et al. 1999).

\section{References}

Atkinson DE \& Fall L (1967) Adenosine triphosphate conservation in biosynthetic regulation. The Journal of Biological Chemistry 10, 3241-3242.

Atkinson DE \& Walton GM (1967) Adenosine triphosphate conservation in metabolic regulation. The Journal of Biological Chemistry 10, 3239-3241.

Aulwurm UR \& Brand KA (2000) Increased formation of reactive oxygen species due to glucose depletion in primary cultures of rat thymocytes inhibits proliferation. European Journal of Biochemistry 267, 5693-5698.

Bereiter-Hahn J, Münnich A \& Woiteneck P (1998) Dependence of energy metabolism on the density of cells in culture. Cell Structure and Function 23, 85-93.

Bond CJ, Jurica MS, Mesecar A \& Stoddard BL (2000) Determinants of allosteric activation of yeast pyruvate kinase and identification of novel effectors using computational screening. Biochemistry 39, 15333-15343.

Boros LG, Puigjaner J, Cascante M, Lee WNP, Brandes JL, Bassilian S, Yusuf FI, Williams RD, Muscarella P, Melvin WS \& Schirmer WJ (1997) Oxythiamine and dehydroepiandrosterone inhibit the nonoxidative synthesis of ribose and tumor cell proliferation. Cancer Research 57, 4242-4248.

Boscá L \& Corredor C (1984) Is phosphofructokinase the rate limiting step of glycolysis? Trends in Biochemical Sciences $\mathbf{9}$, 372-373.

Brand K, von Hintzenstern J, Langer K \& Fekl W (1987) Pathways of glutamine and glutamate metabolism in resting and proliferating rat thymocytes: Comparison between free and peptide-bound glutamine. Journal of Cellular Physiology 132, 559-564.

Brazill DT, Thorner J \& Martin S (1997) Mck1, a member of the glycogen synthase kinase 3 family of protein kinases, is a negative regulator of pyruvate kinase in the yeast Saccharomyces cerevisiae. Journal of Bacteriology 179, 4415-4418.

Chesney J, Mitchell R, Benigni F, Bacher M, Spiegel L, Al-Abed Y, Han JH, Metz C \& Bucala R (1999) An inducible gene product for 6-phosphofructo-2-kinase with an AU-rich instability element: role in tumor cell glycolysis and the Warburg effect. Proceedings of the National Academy of Sciences USA 96, 3047-3052.

Chopade BA, Shankar S, Sundin GW, Mukhopadhyay S \& Chakrabarty AM (1997) Characterization of membraneassciated Pseudomonas aeruginosa ras-like protein Pra, a GTP-binding protein that forms complexes with truncated nucleoside diphosphate kinase and pyruvate kinase to modulate GTP synthesis. Journal of Bacteriology 179, 2181-2188.

Durante P, Gueuning MA, Darville MI, Hue L \& Rousseau GG (1999) Apoptosis induced by growth factor withdrawal in fibroblasts overproducing fructose 2,6-bisphosphate. FEBS Letters 448, 239-243.
Eck HP, Drings P \& Dröge W (1989) Plasma glutamate levels, lymphocyte reactivity and death rate in patients with bronchial carcinoma. Journal of Cancer Research and Clinical Oncology 115, 571-574.

Eigenbrodt E, Fister P \& Reinacher M (1985) New perspectives on carbohydrate metabolism in tumor cells. In Regulation of Carbohydrate Metabolism, pp. 141-179 [R Beitner, editor]. Boca Raton, FL: CRC Press.

Eigenbrodt E, Reinacher M, Scheefers-Borchel U, Scheefers H \& Friis RR (1992) A double role for pyruvate kinase type M2 in the expansion of phosphometabolite pools found in tumor cells. In Critical Reviews in Oncogenesis, pp. 91-115 [M Perucho, editor]. Boca Raton, FL: CRC Press.

Eigenbrodt E, Gerbracht U, Mazurek S, Presek P \& Friis R (1994) Carbohydrate metabolism and neoplasia: New perspectives for diagnosis and therapy. In Biochemical and Molecular Aspects of Selected Cancers, pp. 311-381 [TG Pretlow and TP Pretlow, editors]. San Diego: Academic Press volume 2.

Eigenbrodt E, Kallinowski F, Ott M, Mazurek S \& Vaupel P (1998a) Pyruvate kinase and the interaction of amino acid and carbohydrate metabolism in solid tumors. Anticancer Research 18, 3267-3274.

Eigenbrodt E, Mazurek S \& Friis RR (1998) Double role of pyruvate kinase type $\mathrm{M} 2$ in the regulation of phosphometabolite pools. In Cell Growth and Oncogenesis, pp. 15-30 [P Bannasch, D Kanduc, S Papa and JM Tager, editors]. Basel, Switzerland: Birkhäuser Verlag.

Engel M, Seifert M, Theisinger B, Seyfert U \& Welter C (1998) Glyceraldehyde 3-phosphate dehydrogenase and Nm23-H1/nucleoside diphosphate kinase A. The Journal of Biological Chemistry 273, 20058-20065.

Gosalvez M, Pérez-García J \& Weinhouse S (1974) Competition for ADP between pyruvate kinase and mitochondrial oxidative phosphorylation as a control mechanism in glycolysis. European Journal of Biochemistry 46, 133-140.

Grimm H, Tibell A, Norrlind B, Blecher C, Wilker S \& Schwemmle K (1994) Immunoregulation by parenteral lipids: impact of the n-3 to n-6 fatty acid ratio. Journal of Parenteral and Enteral Nutrition 18, 417-421.

Grimm H, Tibell A, Norrlind B, Schott J \& Bohle RM (1995) Nutrition and allorejection impact of lipids. Transplant Immunology 3, 62-67.

Hess B (1963) Control of metabolic rates. In Control in Respiration and Fermentation, pp. 333-350 [B Hess and B Wright, editors]. New York: Ronald Press.

Jiang WG, Bryce RP \& Horrobin DF (1998) Essential fatty acids: molecular and cellular basis of their anti-cancer action and clinical implications. Critical Reviews in Oncology/Hematology 27, 179-209.

Kallinowski F, Fortmeyer HP, Runkel S \& Vaupel P (1987) Substrate utilization of human tumor xenografts in vivo. Journal of Nutritional Growth Cancer 4, 155-166.

Kallinowski F, Vaupel P, Runkel S, Berg G, Fortmeyer HP, Baessler KH \& Wagner K (1988) Glucose uptake, lactate release, ketone body turnover, metabolic micromilieu, and $\mathrm{pH}$ distributions in human breast cancer xenografts in nude rats. Cancer Research 48, 7264-7272.

Kew S, Wells SM, Yaqoob P, Wallace FA, Miles EA \& Calder PC (1999) Dietary glutamine enhances murine T-lymphocyte responsiveness. Journal of Nutrition 129, 1524-1531.

Lee WNP, Byerley LO, Bassilian S, Ajie HO, Clark I, Edmond J \& Bergner EA (1995) Isotopomer study of lipogenesis in human hepatoma cells in culture: contribution of carbon and reducing hydrogen from glucose. Analytica Biochemica 226, $100-112$.

Lee WNP, Boros LG, Puigjaner J, Bassilian S, Lim S \& Cascante M (1998) Mass isotopomer study of the nonoxidative pathways 
of the pentose cycle with [1,2-13C 2 glucose. American Journal of Physiology 274, E843-E851.

Live TR \& Kaminskas E (1975) Changes in adenylate energy charge in Ehrlich ascites tumor cells deprived of serum, glucose, or amino acids. The Journal of Biological Chemistry 250, 1786-1789.

Luesink EJ, Beumer CMA, Kuipers OP \& De Vos WM (1999) Molecular characterization of the lactococcus lactis ptsH1 operon and analysis of the regulatory role of HPr. Journal of Bacteriology 181, 764-771.

Maeba P \& Sanwal BD (1968) The regulation of pyruvate kinase of Escherichia coli by fructose diphosphate and adenylic acid. The Journal of Biological Chemistry 243, 448-450.

Marchut E, Gumínska M \& Ķdryna T (1986) The inhibitory effect of various fatty acids on aerobic glycolysis in Ehrlich ascites tumour cells. Acta Biochimica Polonica 33, 7-16.

Mazurek S, Hugo F, Failing K \& Eigenbrodt E (1996) Studies on associations of glycolytic and glutaminolytic enzymes in MCF-7 cells: role of p36. Journal of Cellular Physiology 167, 238-250.

Mazurek S, Boschek CB \& Eigenbrodt E (1997a) The role of phosphometabolites in cell proliferation, energy metabolism, and tumor therapy. Journal of Bioenergetics and Biomembranes 29, 315-330.

Mazurek S, Michel A \& Eigenbrodt E (1997b) Effect of extracellular AMP on cell proliferation and metabolism of breast cancer cell lines with high and low glycolytic rates. The Journal of Biological Chemistry 272, 4941-4952.

Mazurek S, Grimm H, Wilker S, Leib S \& Eigenbrodt E (1998) Metabolic characteristics of different malignant cancer cell lines. Anticancer Research 18, 3275-3282.

Mazurek S, Weise G, Wüst G, Schäfer-Schwebel A, Eigenbrodt E \& Friis RR (1999a) Energy metabolism in the involuting mammary gland. in vivo 13, 467-478.

Mazurek S, Eigenbrodt E, Failing K \& Steinberg P (1999b) Alterations in the glycolytic and glutaminolytic pathways after malignant transformation of rat liver oval cells. Journal of Cellular Physiology 181, 136-146.

Mazurek S, Grimm H, Oehmke M, Weisse G, Teigelkamp S \& Eigenbrodt E (2000) Tumor M2-PK and glutaminolytic enzymes in the metabolic shift of tumor cells. Anticancer Research 20, 5151-5154.

Mazurek S, Zwerschke W, Jansen-Dürr P \& Eigenbrodt E (2001) Effects of the HPV-16 E7 oncoprotein on glycolysis and glutaminolysis: Role of pyruvate kinase type M2 and the glycolytic enzyme complex. Biochemical Journal, 356, 247-256.

McKeehan WL (1982) Glycolysis, glutaminolysis and cell proliferation. Cell Biology International Reports 6, 635-650.

Melo RF, Stevan FR, Campello AP, Carnieri EGS \& Martinelli de Oliveira MB (1998) Occurrence of the crabtree effect in Hela cells. Cell Biochemistry and Function 16, 99-105.

Nelson DL \& Kornberg A (1970a) Biochemical studies of bacterial sporulation and germination. Phosphate metabolism during sporulation. The Journal of Biological Chemistry 245, 1137-1145.

Nelson DL \& Kornberg A (1970b) Biochemical studies of bacterial sporulation and germination. Phosphate metabolism during germination. The Journal of Biological Chemistry 245, 1146-1155.

Newsholme EA \& Calder PC (1997) The proposed role of glutamine in some cells of the immune system and speculative consequences for the whole animal. Nutrition 13, 728-730.

Nguyen TN, Wang HJ, Zalzal S, Nanci A \& Nabi IR (2000) Purification and characterization of beta-actin-rich tumor cell pseudopodia: role of glycolysis. Experimental Cell Research 258, $171-183$.

Oremek GM, Teigelkamp S, Kramer W, Eigenbrodt E \& Usadel $\mathrm{K}-\mathrm{H}$ (1999) The pyruvate kinase isoenzyme tumor M2 (Tu
M2-PK) as a tumor marker for renal carcinoma. Anticancer Research 19, 2599-2602.

Perez JX, Roig T, Manzano A, Dalmau M, Boada J, Ventura F, Rosa JL, Bermudez J \& Bartrons R (2000) Overexpression of fructose 2,6-bisphosphatase decreases glycolysis and delays cell cycle progression. American Journal of Physiological Cell Physiology 279, C1359-C1365.

Pizer ES, Chrest FJ, DiGiuseppe JA \& Han WF (1998) Pharmacological inhibitors of mammalian fatty acid synthase suppress DNA replication and induce apoptosis in tumor cell lines. Cancer Research 58, 4611-4615.

Popanda O, Fox G \& Thielmann HW (1998) Modulation of DNA polymerases $\alpha, \delta$ and $\varepsilon$ by lactate dehydrogenase and 3-phosphoglycerate kinase. Biochimica et Biophysica Acta 1397, 102-117.

Presek P, Reinacher M \& Eigenbrodt E (1988) Pyruvate kinase type M2 is phosphorylated at tyrosine residues in cells transformed by Rous sarcoma virus. FEBS Letters 242, 194-198.

Punnonen K, Hietanen E, Auvinen O \& Punnonen R (1989) Phospholipids and fatty acids in breast cancer tissue. Journal of Cancer Research and Clinical Oncology 115, 575-578.

Rhaese H-J, Grade R \& Dichtelmüller H (1976) Studies on the control of development. European Journal of Biochemistry 64, 205-213.

Schramm A, Siebers B, Tjaden B, Brinkmann H \& Hensel R (2000) Pyruvate kinase of the hyperthermophilic Crenarchaeote Thermoproteus tenax: Physiological role and phylogenetic aspects. Journal of Bacteriology 182, 2001-2009.

Sebolt JS \& Weber G (1984) Negative correlation of L-glutamine concentration with proliferation rate in rat hepatomas. Life Sciences 34, 301-306.

Shim H, Chun YS, Lewis BC \& Dang CV (1998) A unique glucose dependent apoptotic pathway induced by c-Myc. Proceedings of the National Academy of Sciences, USA 95, 1511-1516.

Teusink B, Walsh MC, van Dam K \& Westerhoff HV (1998) The danger of metabolic pathways with turbo design. Trends in Biochemical Sciences 23, 162-169.

Thevelein JM \& Hohmann S (1995) Trehalose synthase: guard to the gate of glycolysis in yeast? Trends in Biochemical Sciences 20, 3-10.

Thomas S \& Fell DA (1998) A control analysis exploration of the role of ATP utilisation in glycolytic-flux control and glycolytic metabolite concentration regulation. European Journal of Biochemistry 258, 956-967.

Tuominen FW \& Bernlohr RW (1971) Pyruvate kinase of the spore-forming bacterium, Bacillus licheniformis. The Journal of Biological Chemistry 246, 1746-1755.

Valle F, Muňoz E, Ponce E, Flores N \& Bolivar F (1996) Basic and applied aspects of metabolic diversity: the phosphoenolpyruvate node. Journal of Industrial Microbiology 17, 458-462.

Vaupel P, Fortmeyer HP, Runkel S \& Kallinowski F (1987) Blood flow, oxygen consumption, and tissue oxygenation of human breast cancer xenografts in nude rats. Cancer Research 47, 3496-3503.

Wang H \& Iynedjian PB (1997) Acute glucose intolerance in insulinoma cells with unbalanced overexpression of glucokinase. The Journal of Biological Chemistry 272, 25731-25736.

Wheeler MD, Ikejema K, Enomoto N, Stacklewitz RF, Seabra V, Zhong Z, Yin M, Schemmer P, Rose ML, Rusyn I, Bradfprd B \& Thurman RG (1999) Glycine: a new anti-inflammatory immunonutrient. Cellular Molecular Life Sciences 56, 843-856.

Zwerschke W, Mazurek S, Massimi P, Banks L, Eigenbrodt E \& Jansen-Dürr P (1999) Modulation of type M2 pyruvate kinase activity by the human papillomavirus type 16 E7 oncoprotein. Proceedings of the National Academy of Sciences, USA 96, $1291-1296$ 